\title{
A Comparison of Fever Classified Chief Complaints and Diagnoses with Recorded Body Temperatures
}

\author{
Patricia Araki*, Emily Kajita, Kelsey OYong, Monica Z. Luarca, Bessie Hwang and Laurene \\ Mascola
}

County of Los Angeles, Department of Public Health, Los Angeles, CA, USA

\section{Objective}

The Los Angeles County (LAC) Emergency Department (ED) Syndromic Surveillance System (SSS) classifies patients into syndrome categories based on stated chief complaints. In an effort to evaluate the accuracy of patient- stated chief complaints and final diagnoses, both "fever" chief complaints and diagnoses were compared with patient body temperature readings.

\section{Introduction}

The LAC SSS has been in existence since 2004. Currently, the system collects data from over 50 hospitals daily and performs a chief complaint-based syndrome classification analysis of all ED visits. The keyword "fever" is of special interest due to its inclusion within several syndrome category definitions such as influenza, meningitis, etc. However, inclusion of such terms in syndrome definitions may be a disadvantage as such keyword searches would depend upon the consistency in which the term "fever" is reported. In 2014, several LAC syndromic surveillance hospital data connections were upgraded to include notes recording patient body temperature. To evaluate the newly added temperature information, analyses were conducted on those observations that included body temperature, chief complaint, and diagnosis information.

\section{Methods}

For this study, emergency department admitting data from 9 hospitals were reviewed. A total of 24,402 observations from a five month period were categorized into groups by patient body temperature, those with fever classified chief complaints $(n=1441)$ that included the terms "fever" or "febrile", or patients with a similarly defined fever classified diagnosis ( $\mathrm{n}=970)$ and/or corresponding ICD9 code. Binary classification tests were conducted on this population to observe fever classified chief complaint and diagnosis outcomes against selected body temperature ranges.

\section{Results}

The sensitivities for fever classified chief complaints were $28.5 \%$, $59.8 \%$, and $73.9 \%$ for body temperatures $\geq 99^{\circ} \mathrm{F}, \geq 100^{\circ} \mathrm{F}$, and $\geq 101^{\circ} \mathrm{F}$, respectively. Corresponding positive predictive values (PPV) were $60.7 \%, 43.2 \%$, and $27.8 \%$ for the same groups. For fever classified diagnoses, sensitivities were found to be $22.2 \%, 47.5 \%$, and $56.9 \%$ while PPVs were at $70.3 \%, 51.0 \%$, and $31.8 \%$, respectively, for the same temperature groups. The majority of fever classified chief complaints $(81.4 \%)$ and diagnoses $(75.7 \%)$ were categorized into the respiratory syndrome category with $54.3 \%$ of all fever classified chief complaints resulting in a consistent diagnosis. Furthermore, $55 \%$ of observations with both a fever classified chief complaint and diagnosis also recorded body temperatures at or above $100^{\circ} \mathrm{F}$.

\section{Conclusions}

These preliminary findings have provided a basic understanding of the utilization of the term "fever" within the LAC SSS. In addition, the evaluation of body temperature versus fever classified chief complaints and diagnoses will be utilized to determine whether, and how, the body temperature variable may be included in future iterations of syndrome classification algorithms.

\section{Keywords}

fever; syndromic surveillance; temperature; chief complaint

\section{*Patricia Araki}

E-mail:paraki@ph.lacounty.gov 